\title{
LOCALIZED MODES IN A CIRCULAR ARRAY OF COUPLED NONLINEAR OPTICAL WAVEGUIDES
}

\author{
K. HIZANIDIS*, S. DROULIAS and I. TSOPELAS \\ School of Electrical and Computer Engineering, \\ National Technical University of Athens, Athens 157 73, Greece \\ *kyriakos@central.ntua.gr \\ N. K. EFREMIDIS and D. N. CHRISTODOULIDES \\ School of Optics/CREOL, University of Central Florida, \\ Orland, FL 32816-2700, USA
}

Received January 13, 2005; Revised April 12, 2005

\begin{abstract}
A circular array of optical waveguides collectively coupled with a central core is investigated. Nonlinear losses, both linear and nonlinear coupling as well as energy transfer between neighboring array elements and between the array and the core are allowed. The concept is ideal for the design of high power stable amplifiers as well as of all-optical data processing devices in optical communications. The existence of stable steady-state continuous wave modes as well as of localized solitary and breathing type modes is demonstrated. These properties render the proposed system functionally rich, far more controllable than a planar one and easier to stabilize.
\end{abstract}

Keywords: Breathers; solitons; optical waveguide arrays.

\section{Introduction}

The continuous complex Ginzburg-Landau (cCGL) equation is well known as the dominant underlying model in superconductivity, superfluidity, nonequilibrium fluid dynamics, physical chemistry, nonlinear optics, Bose-Einstein condensates, quantum field theories [Cross \& Hohenberg, 1993; Kuramoto, 1984; Aranson \& Kramer, 2002; Akhemediev \& Ankiewitz, 1977; Manneville, 1990]. On the other hand, the discrete complex Ginzburg-Landau (dCGL) have also been considered in lattices for modeling vortices in hydrodynamics [Willaime et al., 1991] as well as the dynamics of an open Bose-Einstein condensate (where dissipation is naturally expected) with a lattice potential created by the interference of two standing optical waves [Anderson \& Kasevich, 1998] and a gain resulting from the interaction among condensed and uncondensed atoms [Kneer et al., 1998; Arecchi et al., 2002]. As far as the applied optics is concerned the discrete Nonlinear Schrödinger equation (dNLS) was first introduced as a working model in semiconductor laser arrays in optics [Wang \& Winful, 1988; Christodoulides \& Joseph, 1988; Otsuka, 1999; Schmidt-Hattenberger et al., 1991; Eisenberg et al., 1998]. More recently, the dCGL takes the lead in several works [Efremidis \& Hizanidis, 2002; Efremidis et al., 2002; Efremidis \& Christodoulides, 2003; Maruno et al., 2003]: It is worth mentioning that while in cCGL equation-based systems, selflocalized (solitary) solutions as well as dissipative solitons have been found [Pereira \& Stenflo, 1977; Nozaki \& Bekki, 1984; Hocking \& Stewartson, 1992] (among an extraordinary universe of pattern formation and chaotic behavior), no coherent structures have been found in dCGL equation-based systems until very recently [Efremidis \& Christodoulides, 2003; Maruno et al., 2003].

Although the underlying physics in a dCGL equation-based system is complex and not easily 
amenable to analytical considerations, the applications are potentially far reaching. In this paper the main focus is on the design of high power stable amplifiers as well as of all-optical data processing devices in optical communications where studying the stability, phase-locking capabilities and rich controllable behavior of the nonlinear modes during evolution is of great importance. The concept is based on an array of optical waveguides, where both linear and nonlinear coupling and energy transfer may enrich the functionality of such a device. It is expected that the analysis of such devices will become quite expansive especially in geometries beyond planar, such as circular [SchmidtHattenberger et al., 1991] which is the subject of this work. In addition to the circular geometry, the waveguide array is linearly and nonlinearly coupled to a central core allowing energy transfer and exchange not only among the various array elements but also collectively with the central core. It is envisioned that this collective coupling will render the whole system far more controllable than a planar one and easier to stabilize. There are numerous indications in the literature (concerning cCGL, however) which are in support of this conjecture [Efremidis \& Hizanidis, 2002; Malomed \& Winful, 1984].

The paper is organized as follows: Section 2 discusses the model equations and conditions for linear stability. In Sec. 3 the existence of stable nonlinear CW solutions is investigated, while in Sec. 4, the generation mechanism of breathing and solitarytype solutions is presented. The main conclusions are summarized in Sec. 5.

\section{The Model and Conditions for Linear Stability}

The system under investigation consists of a circular array of $N$ identical linearly and nonlinearly coupled (with the nearest neighbors) optical waveguides (active and/or lossy optical fibers, for instance). The array is also linearly as well as nonlinearly coupled (cubic nonlinearity) with a cylindrical substrate (central core). The whole system is embedded in a cladding of infinite extent. We allow for complex linear coupling coefficients between each node of the array and its neighbors as well as for lossy and/or amplifying behavior in each node of the array separately and in the central core. The reference signs of the imaginary parts of all the linear coupling coefficients involved are chosen in such a way that if they all were positive the model would then represent a circular array of optical amplifiers coupled with a lossy central core. The model can equally well describe a modification of a planar semiconductor laser array [Otsuka, 1999] to a circular one (away from the saturation limit of the lasing medium) lying on a dielectric cylindrical substrate.

By using the formalism of coupled-mode theory (or the tight binding approximation) [Otsuka, 1999; Kittel, 1986] one obtains the following equations in the framework of slowly-varying envelope approximation for the discrete complex mode amplitude $\left\{u_{n}(z), n=1, \ldots, N ; u_{0}(z)\right\}$ with $z$ being the propagation distance and " 0 " referring to the central core,

$$
\begin{aligned}
& i \frac{d u_{n}}{d z}-i \varepsilon g\left(I_{n}\right) u_{n}+\mu u_{n}\left|u_{n}\right|^{2}+\kappa_{\alpha}\left(u_{n+1}+u_{n-1}\right) \\
& \quad-i \varepsilon_{\alpha} g\left(I_{n}\right)\left(u_{n+1}+u_{n-1}\right) \\
& \quad+\kappa_{X} u_{0}-i \varepsilon_{X} G\left(I_{0}\right) u_{0}=0 \\
& i \frac{d u_{0}}{d z}-i E G\left(I_{0}\right) u_{0}+M\left|u_{0}\right|^{2} u_{0} \\
& \quad+\kappa_{X} \sum_{n=1}^{N} u_{n}-i \varepsilon_{X} \sum_{n=1}^{N} g\left(I_{n}\right) u_{n}=0
\end{aligned}
$$

where $\varepsilon$ and $E$ are the respective amplification rates for the array elements and the core while $\varepsilon_{\alpha}$ is the gain due to coupling with the nearest neighbors (note that $\varepsilon<0, E<0$ and $\varepsilon_{\alpha}<0$ represent lossy nodes, lossy central core and lossy coupling, respectively). The factors $g(I), G(I)$ are the respective normalized gains expressed as functions of the normalized intensity $I=|u|^{2}$, while $\mu$ and $M$ are the respective self-phase modulation coefficients. Furthermore $a$ and $A$ are the respective nonlinear loss coefficients and $\kappa_{\alpha}$ the linear coupling constant with the nearest neighbors. Finally, $\kappa_{X}$ is the linear coupling between the central core and the array elements and $\varepsilon_{X}$ models the gain due to linear coupling between the central core and the array elements. For intensity values below the saturation limit, these gain functions acquire values below but not far from unity and can be linearly approximated by [Desurvire, 1994],

$$
g\left(I_{n}\right) \approx 1-\alpha I_{n}, \quad G\left(I_{0}\right) \approx 1-A I_{0} .
$$

The system of Eqs. (1) and (2) can be further simplified since the energy tunneling, expressed by the constants $\varepsilon_{\alpha}$ and $\varepsilon_{X}$ is a small effect compared with 
the effects modeled by $\varepsilon$ and $E$. Thus, the respective intensity-dependent contributions associated with the energy tunneling are dropped ( $\alpha$ and $A$ are small) yielding,

$$
\begin{aligned}
& i \frac{d u_{n}}{d z}-i \varepsilon u_{n}+(\mu+i \alpha) u_{n}\left|u_{n}\right|^{2} \\
& \quad+\left(\kappa_{\alpha}-i \varepsilon_{\alpha}\right)\left(u_{n+1}+u_{n-1}\right) \\
& \quad+\left(\kappa_{X}-i \varepsilon_{X}\right) u_{0}=0, \\
& i \frac{d u_{0}}{d z}-i E u_{0}+(M+i A)\left|u_{0}\right|^{2} u_{0} \\
& \quad+\left(\kappa_{X}-i \varepsilon_{X}\right) \sum_{n=1}^{N} u_{n}=0
\end{aligned}
$$

The simplified system has the form of a cubic dCGL coupled with a discrete nonlinear Schrödinger (dNLS) equation.

In order to investigate the stability of the zero solution, one may choose to investigate the linear approximation of Eqs. (4) and (5). The stability of the zero solution is of great importance in the investigation of the existence of stable localized modes in open systems, since then the optical power is concentrated in a subset of nodes leading to solitary and/or breather type of structures. The reason is that localized structures residing on a linear array have their intensity dropping to zero at their tails, that is, at the open ends of the array. For the circular system in hand this is not the case since the tails coincide. This will become evident shortly when the comparison of regions of linear and nonlinear stability will be made. However, in the following, we proceed in briefly investigating the linear stability for comparison.

The centrally uncoupled version of the linear approximation of the model $\left(\kappa_{\chi}-i \varepsilon_{\chi}=\mu+i \alpha=\right.$ $M+i A=0)$ is a Toeplitz type of a problem with known eigenmode structure [Schmidt-Hattenberger et al., 1991]. In the coupled case of the same approximation the resulting eigenvalue problem is modified. By straightforward algebraic manipulations it can be shown that the first $N-1$ eigenvalues coincide with those in the uncoupled case (Toeplitz problem), that is,

$$
\lambda_{k}=-i \varepsilon+2\left(\kappa_{\alpha}-i \varepsilon_{\alpha}\right) \cos \left(\frac{2 \pi k}{N}\right), \quad k=1, \ldots, N
$$

while the remaining two additional eigenvalues are given by the following expression

$$
\begin{aligned}
\lambda_{N, N+1}= & \kappa_{\alpha}-\frac{i\left(\varepsilon_{T}+E\right)}{2} \\
& \pm \sqrt{N\left(\kappa_{\chi}-i \varepsilon_{\chi}\right)^{2}+\left[\kappa_{\alpha}-\frac{i\left(\varepsilon_{T}-E_{0}\right)}{2}\right]^{2}}
\end{aligned}
$$

where "+" and "-" stand for the $N$ th and $(N+1)$ th eigenvalue respectively and $\varepsilon_{T} \equiv \varepsilon+2 \varepsilon_{\alpha}$. The general linear solution for the array and the central core can easily be expressed as a superposition of normal modes,

$$
\begin{aligned}
u_{n}= & a_{N} u^{(N)} e^{i \lambda_{N} z}+a_{N+1} u^{(N+1)} e^{i \lambda_{N+1} z} \\
& +\sum_{k=1}^{N-1} \frac{a_{k} e^{\frac{i 2 \pi k n}{N+i \lambda_{k} z}}}{\sqrt{N}} \\
u_{0}= & b_{N} U^{(N)} e^{i \lambda_{N} z}+b_{N+1} U^{(N+1)} e^{i \lambda_{N+1} z}
\end{aligned}
$$

and $a_{m}, b_{m}$ are free (specified by the launching conditions) complex coefficients, where the two new pairs of $N$ th and $(N+1)$ th eigenfunctions are given by,

$$
\begin{array}{r}
u^{(j)}=\frac{\lambda_{j}+i E_{0}}{\sqrt{N^{2}\left(\kappa_{\chi}-i \varepsilon_{\chi}\right)^{2}+N\left|\lambda_{j}+i E_{0}\right|^{2}}}, \\
U^{(j)}=\sqrt{\frac{N\left(\kappa_{\chi}-i \varepsilon_{\chi}\right)^{2}}{N\left(\kappa_{\chi}-i \varepsilon_{\chi}\right)^{2}+\left|\lambda_{j}+i \mathrm{E}_{0}\right|^{2}}}, \\
j=N, N+1
\end{array}
$$

Stability of the zero solution requires all $N+1$ eigenvalues to possess positive imaginary parts simultaneously. By separating the real and imaginary parts of the $N+1$ eigenvalues the condition for linear stability becomes,

$$
\begin{array}{r}
\max \left(\varepsilon_{T}\right) \\
=\left\{\begin{array}{ll}
2 \varepsilon_{\alpha}\left[1-\cos \left(\frac{2 \pi}{N}\right)\right] & \text { for } \varepsilon_{\alpha} \geq 0 \\
2 \varepsilon_{\alpha}\left\{1-\cos \left[\left(\frac{2 \pi}{N}\right)\left\|\frac{N}{2}\right\|\right]\right\} & \text { for } \varepsilon_{\alpha}<0
\end{array},\right. \\
-\frac{\frac{\varepsilon+E}{2}+\varepsilon_{\alpha}}{2} \geq \sqrt{\frac{\sqrt{M^{2}+D^{2}}-D}{2}}
\end{array}
$$

where $M \equiv 2\left[-N \varepsilon_{\chi} \kappa_{\chi}-\kappa_{\alpha}\left((\varepsilon-E) / 2+\varepsilon_{a}\right)\right]$, $D \equiv N\left(\kappa_{\chi}^{2}-\varepsilon_{\chi}^{2}\right)+\kappa_{\alpha}^{2}-\left[(\varepsilon-E) / 2+\varepsilon_{a}\right]^{2}$ and "\| \|" symbolizes the integer part. The first condition in Eq. (10) assures the stability of the first $N-1$ 
modes existing in the uncoupled case as well, while the second one assures the stability of both the remaining modes.

\section{Nonlinear Analysis: Existence and Stability of Continuous-Wave Solutions}

Looking for continuous-wave nonlinear modes $(\mathrm{CW})$, that is, for modes of the same amplitude in every node of the circular array, one may set

$$
\begin{aligned}
& u_{n}=U e^{i \lambda z}, \quad n=1, \ldots, N, \\
& u_{0}=V e^{i \lambda z}
\end{aligned}
$$

in the fully nonlinear model of Eqs. (4) and (5). Since we are seeking for stable CW solutions, the wavenumber $\lambda$ is supposed to be real. The substitution above leads to a system of equations in terms of $\lambda,|V|^{2}, R_{r}, R_{i}$ (where $R=R_{r}+i R_{i}=U / V$ )

$$
\begin{gathered}
\left\{-N\left(\kappa_{X}+\frac{M}{A} \varepsilon_{X}\right) R_{r}-N\left(\varepsilon_{X}-\frac{M}{A} \kappa_{X}\right) R_{i}\right. \\
\left.-\frac{M}{A} E+\frac{\mu}{\alpha} \varepsilon_{T}+2 \kappa_{\alpha}\right\}|R|^{2}+\left(\frac{\mu}{\alpha} \kappa_{X}-\varepsilon_{X}\right) R_{i} \\
+\left(\frac{\mu}{\alpha} \varepsilon_{X}+\kappa_{X}\right) R_{r}=0 \\
\varepsilon_{T}|R|^{2}-\frac{\alpha}{A}\left\{E-N\left(\kappa_{X} R_{i}-\varepsilon_{X} R_{r}\right)\right\}|R|^{4} \\
+\kappa_{X} R_{i}+\varepsilon_{X} R_{r}=0 \\
\lambda=N\left(\kappa_{X} R_{r}+\varepsilon_{X} R_{i}\right) \\
+\frac{M}{A}\left\{E-N\left(\kappa_{X} R_{i}-\varepsilon_{X} R_{r}\right)\right\} \\
|V|^{2}=\frac{E-N\left(\kappa_{X} R_{i}-\varepsilon_{X} R_{r}\right)}{A} \quad(12 \mathrm{c}) \\
R^{2}=R_{r}^{2}+R_{i}^{2}
\end{gathered}
$$

Solving (12a), (12b) in terms of $R_{r}, R_{i}$ and combining the results according to (12e), one obtains a sixth order polynomial equation with constant coefficients in terms of $|R|^{2}$ of the form $f\left(|R|^{2} ; \varepsilon_{0}\right.$, $\left.E_{0}, \mu_{0}, M_{0}, a, A, \kappa_{\alpha}, \varepsilon_{\alpha}, \kappa_{X}, \varepsilon_{X}\right)=0$. With the solutions of this equation known, the rest of the unknowns are calculated thus providing the solutions for $U, V$ and $\lambda$, i.e. the $\mathrm{CW}$ solutions. Fixing the parameters $\mu, M, a, A, \kappa_{\alpha}, \varepsilon_{\alpha}$, (or $\varepsilon$ ), $\kappa_{X}, \varepsilon_{X}$ and leaving $\varepsilon_{T}$ and $E$ to vary, domains of stability for the CW solutions can be obtained.

The character of the stability of these steady state CW solutions is an important issue. For these nonlinear modes to be stable the zero solution does not necessarily need to be stable: One can look for stable steady state CW solutions even in the regimes where the zero solution is unstable (several or all $N+1$ linear modes discussed in the previous section). Since the existence of localized solutions is the aim of this work, we focus on the unstable CW modes under amplitude modulation. One may distinguish between two kinds of localized solutions associated with the existence of such unstable CW modes: breather-type, i.e. localized modes periodic in $z$ (and, possibly azimuthally, in $n$, where $n$ is the node number) and solitary-type, i.e. localized modes without any amplitude modulation in $z$. The unstable CW which has the potential to support such a localized nonlinear mode must, at the same time, exhibit stability under periodic (in $z$ ) perturbations since the localized structures are, in general periodic in $z$. The stability (under amplitude modulation and, also, under periodic perturbations) of the CW solutions of Eqs. (4) and (5) cannot be analytically addressed. Therefore, one may linearize these equations around a particular choice for $|U|$ and $|V|$ taken from the respective bifurcation diagram. For each one of the aforementioned two kinds of perturbation, this procedure renders a respective solvable linear system of $2 N+2$ equations for the $N+1$ (randomly chosen) small perturbations of the respective CWs, $\delta u_{n}(n=$ $1, \ldots, N)$ and $\delta u_{0}$ and their complex conjugates, expressed as a $(2 N+2) \times 1$ column vector $\delta \mathbf{u}\left(z^{(n)}\right)$. In each case, the solution can straightforwardly cast in the form, $\delta \mathbf{u}(z)=\exp (z \mathbf{A}) \circ \delta \mathbf{u}(z=0)$, where A is a $(2 N+2) \times(2 N+2)$ block matrix. The constituents of the matrix $\mathbf{A}$ depend upon the choice of steady state CW, the kind of stability we are looking for and the coupling coefficients. The stability is then obviously related directly to the asymptotic behavior (annihilation as $z \rightarrow \infty$ ) of the respective exponential matrix $\exp (z \mathbf{A})$.

In the following, we adopt the following notation: (a) $\mathrm{S}_{\mathrm{CW}}$ for modulationally stable CWs exist in this region among others; as we already mentioned, these CWs cannot support localized modes; (b) $\mathrm{U}_{\mathrm{CW}} \mathrm{S}_{\mathrm{PS}}$ for modulationally unstable CWs which are stable to periodic perturbations, thus, capable of supporting periodic solutions (PS) in $z$ and/or $n$; (c) U $\mathrm{UPS}_{\mathrm{PS}}$ for unstable CWs to periodic perturbations, thus incapable of supporting stable periodic solutions. As an example, in Figs. 1(a)-1(d) the domains of stability are depicted for the case $\varepsilon_{\alpha}=1$, $\varepsilon_{X}=0.1, \mu=\alpha=M=A=0.1, \kappa_{\alpha}=\kappa_{X}=0.1$ 


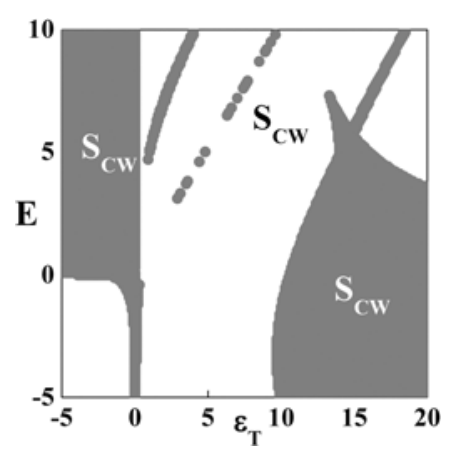

(a)

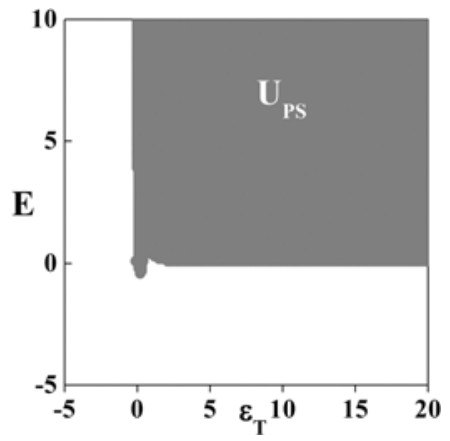

(d)

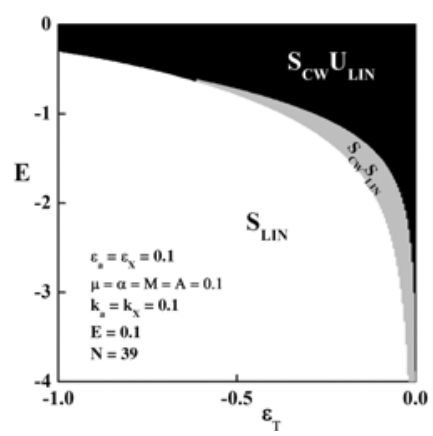

(b)

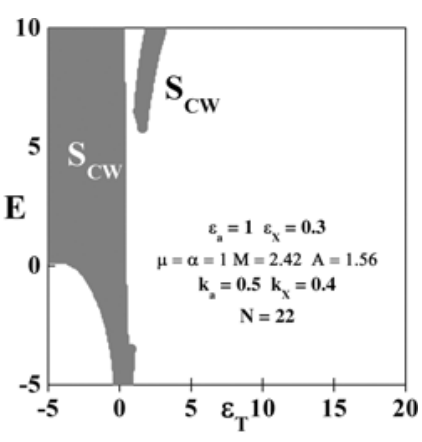

(e)

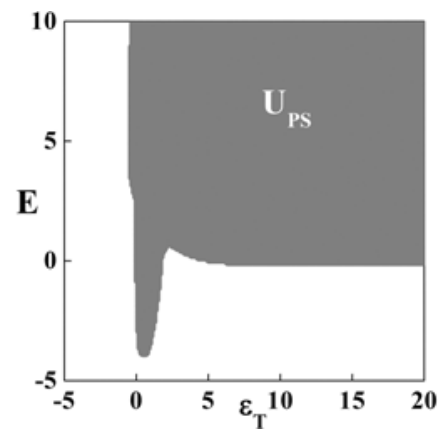

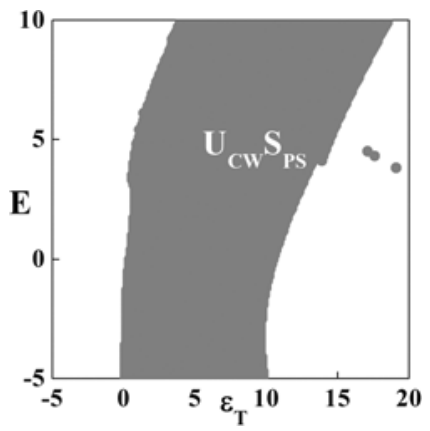

(c)

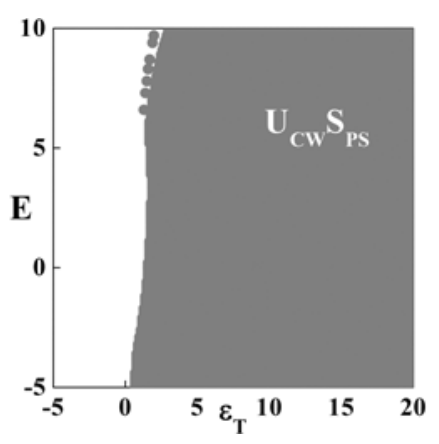

(f)

(g)

Fig. 1. Domains of stability, (a)-(d): $\varepsilon_{\alpha}=1, \varepsilon_{X}=0.1, \mu=\alpha=M=A=0.1, \kappa_{\alpha}=\kappa_{X}=0.1$ and $N=39$; (e)-(g): $\varepsilon_{\alpha}=1$, $\varepsilon_{X}=0.3, \mu=\alpha=1, M=2.42, A=1.56, \kappa_{\alpha}=0.5, \kappa_{X}=0.4$ and $N=22$; (b) is a detail of (a). There are no CW solutions in this domain except from a very narrow layer at the edge (in gray). Notation: $\mathrm{S}_{\mathrm{CW}}$ : modulationally stable $\mathrm{CW}$ exists; $\mathrm{U}_{\mathrm{CW}} \mathrm{S}_{\mathrm{PS}}$ : modulationally unstable $\mathrm{CW}$ which is stable to periodic perturbations exists; $\mathrm{U}_{\mathrm{PS}}$ : unstable CW to periodic perturbations exists.

and $N=39$. Figure 1 (b) provides details of the low left corner of Fig. 1(a) where the linear stability domain resides. There are no CW solutions in this domain except from a very narrow layer at the edge (in gray). In Figs. 1(f) $-1(\mathrm{~g})$ a second example is provided for the case $\varepsilon_{\alpha}=1, \varepsilon_{X}=0.3$, $\mu=\alpha=1, M=2.42, A=1.56, \kappa_{\alpha}=0.5, \kappa_{X}=0.4$ and $N=22$. In conclusion, the parameter space is, by far, dominated and populated by nonlinear CW modes the character of which is illustrated in the two characteristic examples provided in Fig. 1.

Upon a particular choice of the amplification (or, loss) rate $E$ for the central node, bifurcation diagrams can be drawn. In Fig. 2 the respective bifurcation diagrams for the cases of Fig. 1 (Figs. 1(a)-1(c) and Figs. 1(d)-1(e), respectively) 


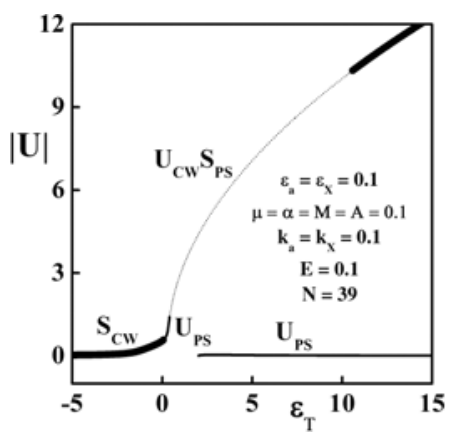

(a)

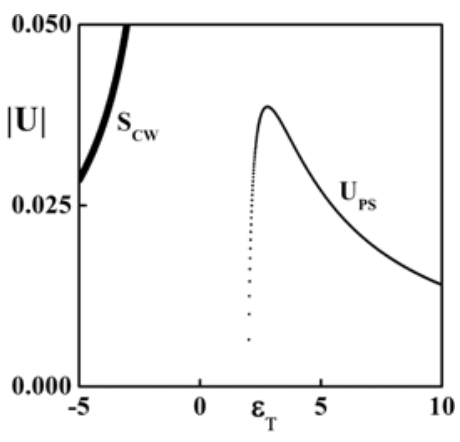

(b)

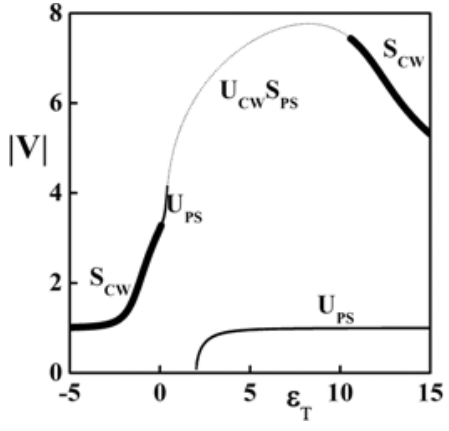

(c)

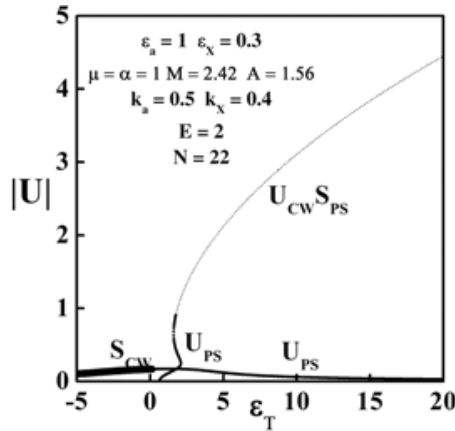

(d)

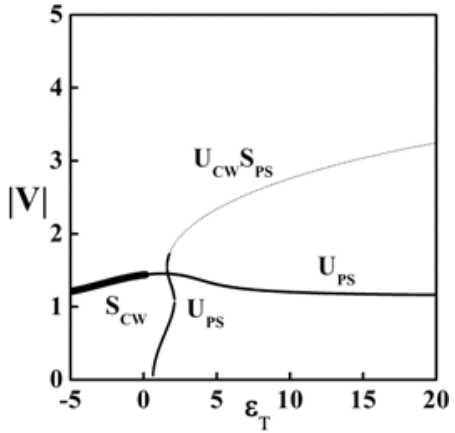

(e)

Fig. 2. Bifurcation diagrams for the CW modes in the array [(a), (b) and (d)] and the central node [(c) and (e)]. (a)-(c): $\varepsilon_{\alpha}=1, \varepsilon_{X}=0.1, \mu=\alpha=M=A=0.1, \kappa_{\alpha}=\kappa_{X}=0.1$ and $N=39 ;(\mathrm{d})-(\mathrm{e}): \varepsilon_{\alpha}=1, \varepsilon_{X}=0.3, \mu=\alpha=1, M=2.42$, $A=1.56, \kappa_{\alpha}=0.5, \kappa_{X}=0.4$ and $N=22$; (b) is a detail of (a).

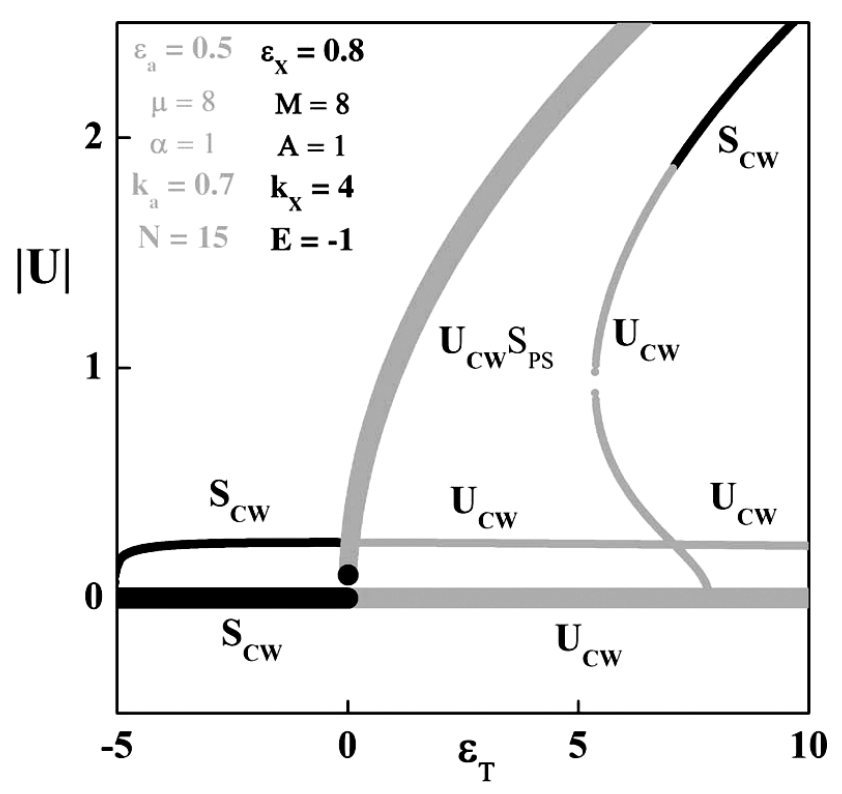

Fig. 3. Comparison of the bifurcations without (thick black lines) and with (thin gray lines) the central node for the case with $N=15, \varepsilon_{\alpha}=0.5, \mu=8, \alpha=1, \kappa_{\alpha}=0.7$ (if the central node is present, then, $\varepsilon_{X}=0.8, M=8, A=1, \kappa_{X}=4$ and $E=-1)$. are shown with respective choices $E=0.1$ and $E=2$. Figure 2(b) is a magnification (near zero amplitude) of Fig. 2(a). In Figs. 2(c) and 2(e) the respective bifurcation diagrams from the perspective of the central node are shown. Finally, in Fig. 3 the comparison of the bifurcations without (thick black lines) and with (thin gray lines) the central node is illustrated for the case with $N=15$, $\varepsilon_{\alpha}=0.5, \mu=8, \alpha=1, \kappa_{\alpha}=0.7$ (if the central node is present, then, $\varepsilon_{X}=0.8, M=8, A=1$, $\kappa_{X}=4$ and $E=-1$ ). It is evident that the presence of the central node introduces multiple $\mathrm{U}_{\mathrm{CW}}$ modes for the same value of $\varepsilon \mathrm{T}$ and an early appearance of $\mathrm{S}_{\mathrm{CW}}$ modes.

\section{In Search of Periodic Nonlinear Modes}

In this section, the generation of periodic nonlinear modes is investigated and discussed. As we already mentioned, from the respective bifurcation diagram and domain of stability we choose a $\mathrm{U}_{\mathrm{CW}} \mathrm{S}_{\mathrm{PS}}$ mode. 
The zero background is obviously linearly unstable as we have already seen.

We first set all nodes of the circular array at $z=0$ to the respective $\mathrm{CW}$ value, while the central node is also set to its respective $\mathrm{CW}$ value. Then, in order to ensure localization (in the node sense), we multiply the array $\mathrm{CW}$ value by an exponential $\exp \left(-\left|n-n_{0}\right|\right)$ or a gaussian $\exp \left[-\left(n-n_{0}\right)^{2}\right]$ filtering factor $\left(n_{0}\right.$ is the node which the filtering discriminates from the rest) which is easily realizable in practical situations. In either case, a periodic mode of breathing type is established after a transition period. In Fig. 4 two cases are shown for both filtering mechanisms. In Figs. 4(a) $-4(\mathrm{~d})$ the choice is $\varepsilon_{\alpha}=1, \varepsilon X=0.3, \mu=M=2.42, A=\alpha=1.56$, $\kappa_{\alpha}=0.5, \kappa_{X}=0.4, E=2, \varepsilon_{T}=2.5$ and $N=83$, while in Figs. 4(e)-4(h) the parameters that differ are $\varepsilon_{T}=2$ and $N=22$. The first column in Fig. 4 provides a three-dimensional view of the amplitude variations axially and azimuthally, while the second column the gray scale equivalent. Figures 4(a), 4(b), 4(e) and 4(f) refer to the exponential filtering factor, while Figs. 4(c), 4(d), 4(g) and 4(h) refer to the gaussian one.

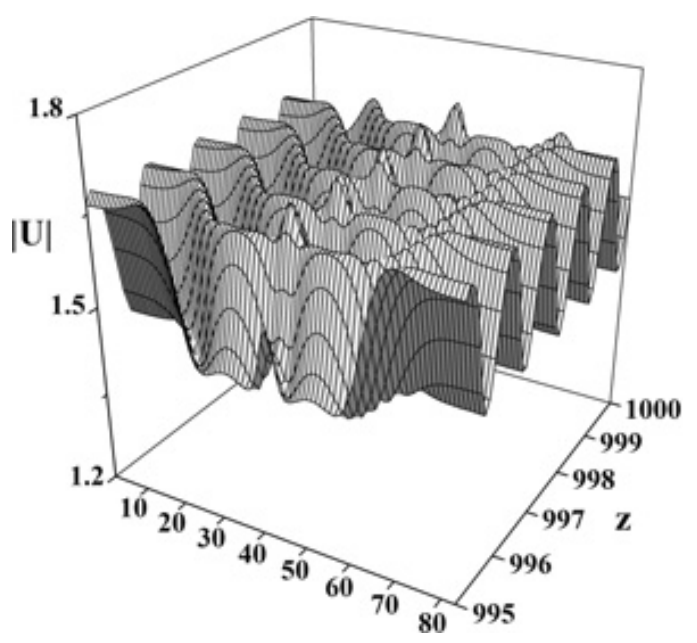

(a)

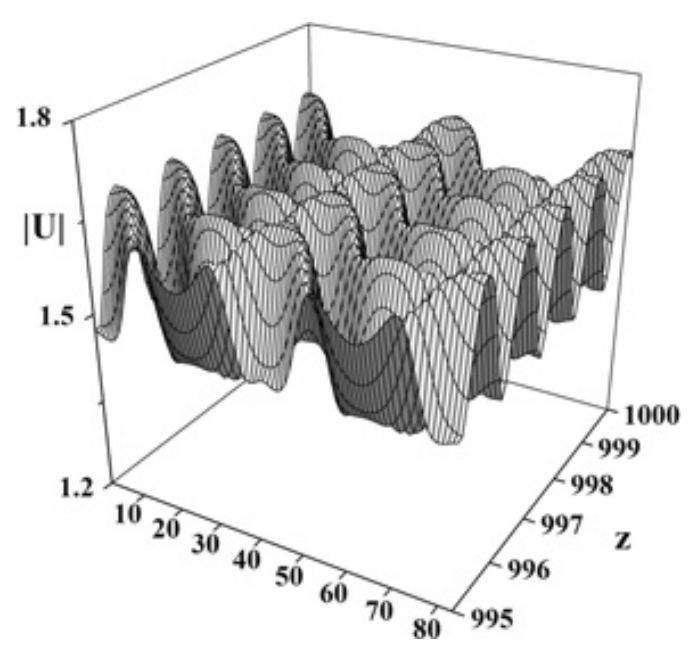

(c)
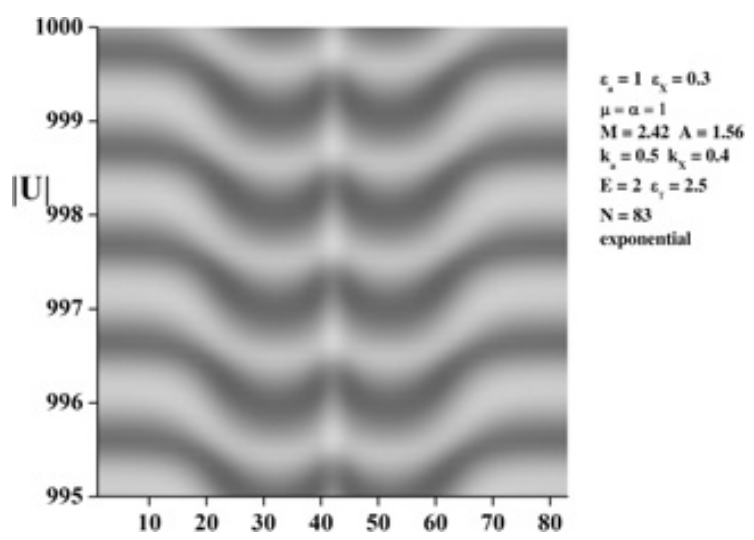

(b)

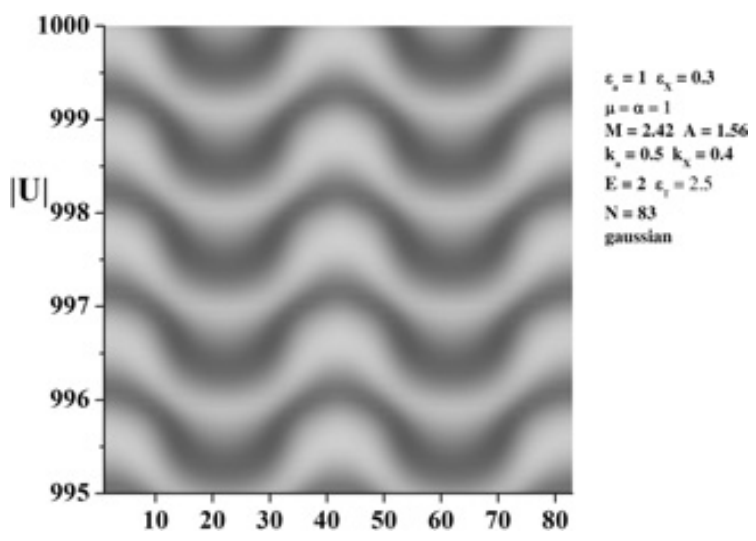

(d)

Fig. 4. $\mathrm{U}_{\mathrm{CW}} \mathrm{S}_{\mathrm{PS}}$ mode (all nodes are set at $z=0$ to their respective $\mathrm{CW}$ values). (a) $-(\mathrm{d}): \varepsilon_{\alpha}=1, \varepsilon_{X}=0.3, \mu=M=2.42$, $A=\alpha=1.56, \kappa_{\alpha}=0.5, \kappa_{X}=0.4, E=2, \varepsilon_{T}=2.5$ and $N=83$; (e)-(h) $\varepsilon_{\alpha}=1, \varepsilon_{X}=0.3, \mu=M=2.42, A=\alpha=1.56$, $\kappa_{\alpha}=0.5, \kappa_{X}=0.4, E=2, \varepsilon_{T}=2$ and $N=22$. The first column provides a three-dimensional view of the amplitude variations axially and azimuthally, while the second column the gray scale equivalent. (a), (b), (e) and (f): exponential filtering factor; (c), (d), (g) and (h): gaussian filtering factor. 


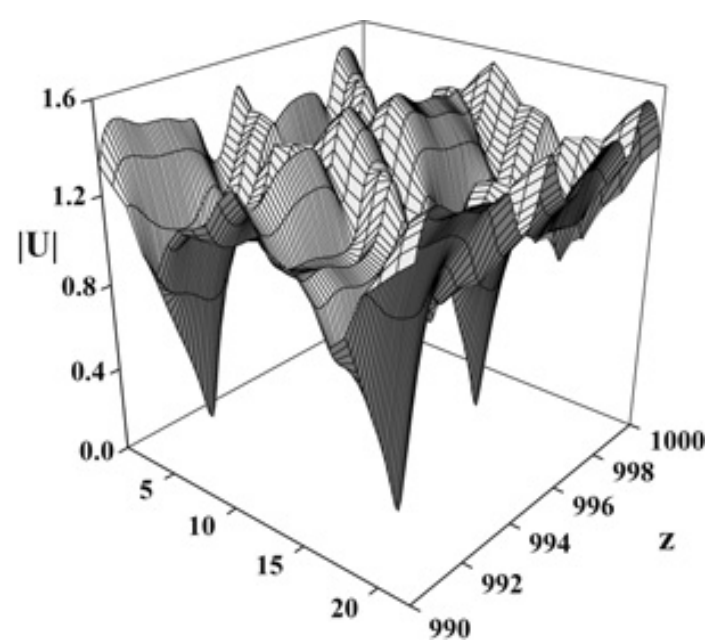

(e)

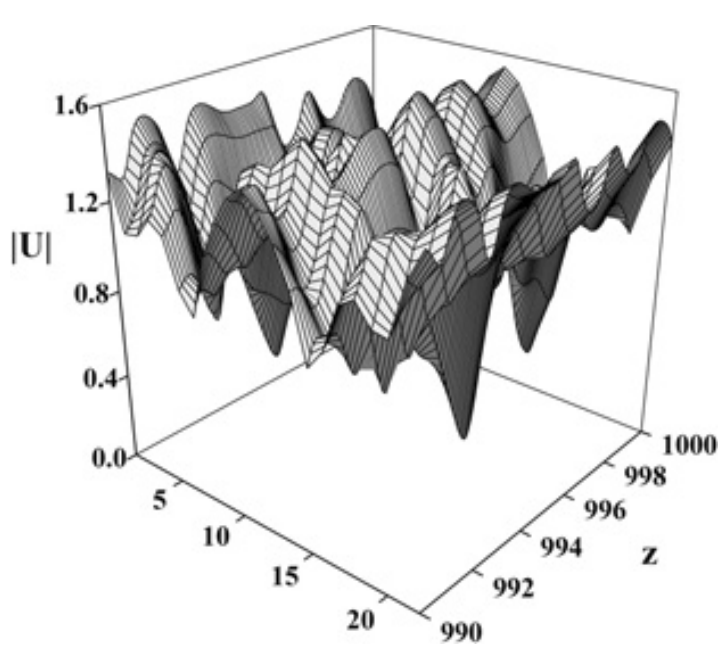

(g)

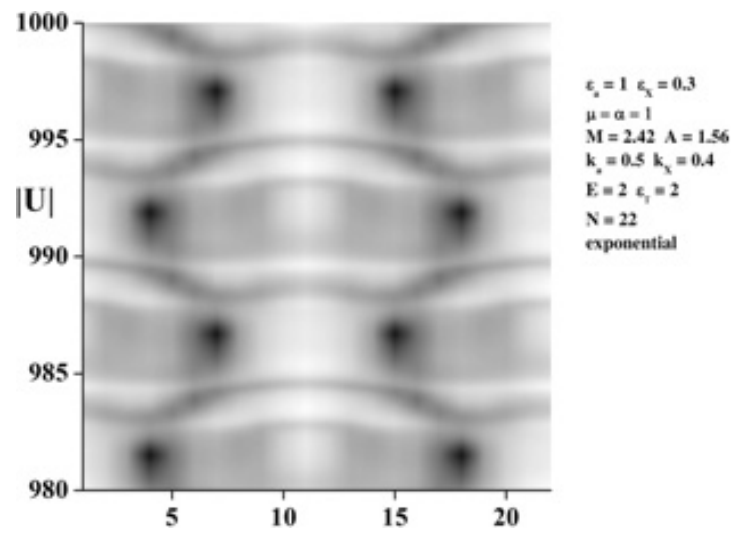

(f)

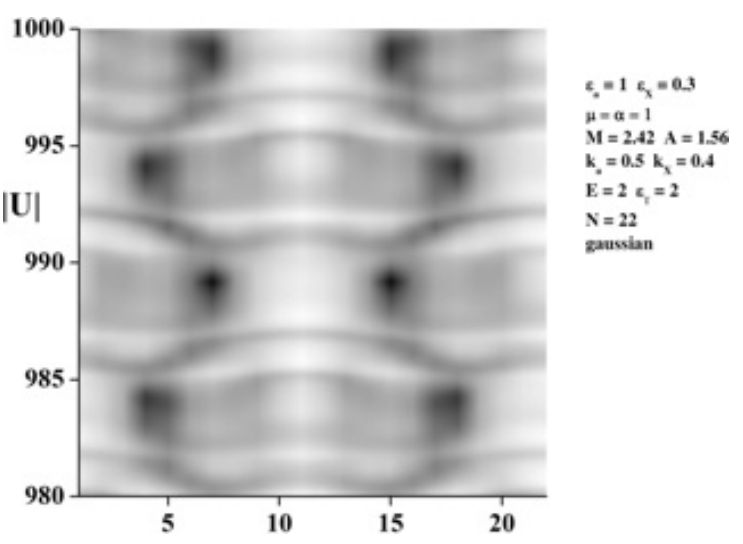

(h)

Fig. 4. (Continued)

An important issue is the behavior of the nonlinear $\mathrm{CW}$ nodes under random perturbations. If the parameters are chosen such as to ensure stability the CW is stable. In such a case one does not expect any periodic nonlinear mode to arise. However, if the parameters are chosen such as to ensure existence of a $\mathrm{U}_{\mathrm{CW}} \mathrm{S}_{\mathrm{PS}}$ mode, then such a mode is expected to arise. The zero background is, of course, linearly unstable in such a case. Setting all nodes of the circular array and the central one at $z=0$ to the respective $\mathrm{CW}$ values and perturbing them randomly (both in amplitude and phase) a periodic mode is indeed established after a transition period. In Fig. 5 an example is shown. The parameter values in this example are $\varepsilon_{\alpha}=1, \varepsilon_{X}=0.1$, $\mu=M=0.1, \alpha=A=0.1, \kappa_{\alpha}=0.1, \kappa_{X}=0.1$,
$E=0.1, \varepsilon_{T}=0.3$ and $N=39$. Figure 5(a) depicts the behavior during the short transition region. Figures 5(b) and 5(c) refer to the asymptotic behavior in a three-dimensional and a gray scale plot, respectively. The asymptotic behavior is reproducible irrespectively of the (random) initial data. Therefore, the unstable $\mathrm{CW}$ nonlinear mode settles to a specific robust and periodic nonlinear mode with both axial and azimuthal variations.

Equally important for practical applications, where control over the light pulse at the launching point is the issue, is the excitation of periodic nonlinear modes by coherent phase modulation of an unstable CW nonlinear mode. In such a case one chooses the parameters such as a $\mathrm{U}_{\mathrm{CW}} \mathrm{S}_{\mathrm{PS}}$ mode to exist. The zero background is again linearly 


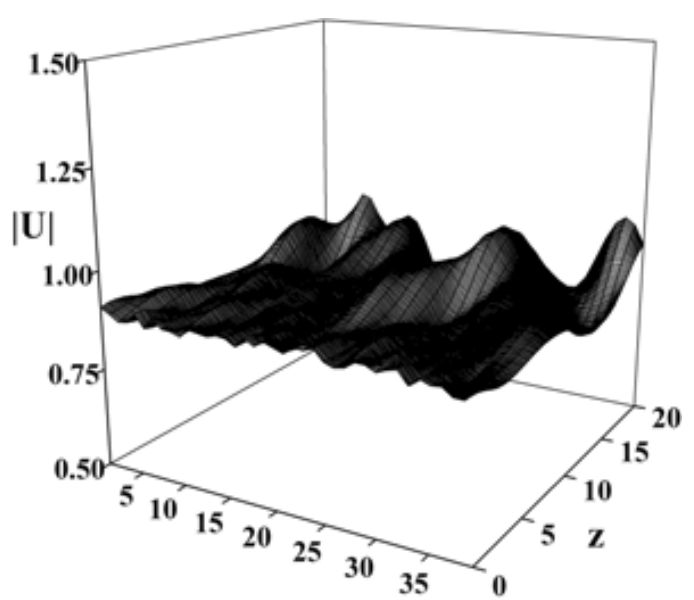

(a)

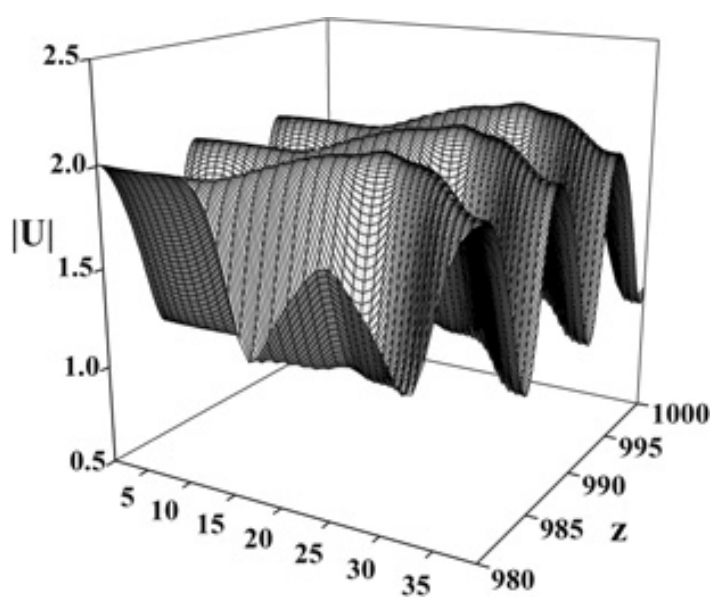

(b)

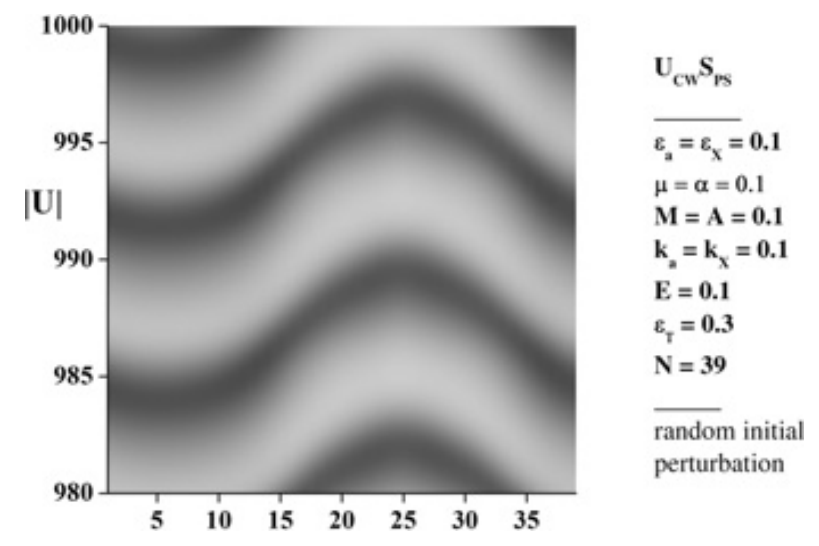

(c)

Fig. 5. $\mathrm{U}_{\mathrm{CW}} \mathrm{S}_{\mathrm{PS}}$ mode (all nodes are set at $z=0$ to their respective $\mathrm{CW}$ values) under random amplitude and phase perturbation with $\varepsilon_{\alpha}=1, \varepsilon_{X}=0.1, \mu=M=0.1, \alpha=A=0.1, \kappa_{\alpha}=0.1, \kappa_{X}=0.1, E=0.1, \varepsilon_{T}=0.3$ and $N=39$. (a) Behavior during the short transition region; (b) three-dimensional plot of the asymptotic behavior; (c) gray scale equivalent of (b).

unstable. Setting all nodes of the circular array and the central one at $z=0$ to the respective $\mathrm{CW}$ values and modulating each node $(n)$ through a phase factor $\exp (j 2 \pi n m / N), m$ being an integer, a periodic mode is established after a short transition period. In the example shown in Fig. 6, the parameters used are $\varepsilon_{\alpha}=1, \varepsilon_{X}=0.1, \mu=M=0.1, \alpha=A=0.1$, $\kappa_{\alpha}=0.1, \kappa_{X}=0.1, E=0.1, \varepsilon_{T}=0.3$ and $N=39$. In Figs. 6(a)-6(c) (short $z$-behavior, asymptotic behavior in three-dimensional and gray scale plot) the azimuthal number $m$ is $m=1$, while in Figs. $6(\mathrm{~d})-6(\mathrm{f}), m=3$. It is evident that the azimuthal number controls the number of nodes where the intensity peaks while the overall light pattern, as it propagates along $z$, rotates azimuthally at the same time.

One may further exploit the bifurcation diagrams in order to access the asymptotic behavior of a nonlinear mode which is excited as a $U_{P S}$ one. We may distinguish between two possible situations: (a) the chosen $\mathrm{CW}$ is a $\mathrm{U}_{\mathrm{PS}}$ mode while a $\mathrm{S}_{\mathrm{CW}}$ of different amplitude exists for the same value of $\varepsilon_{T}$, (b) the chosen $\mathrm{CW}$ is a $\mathrm{U}_{\mathrm{PS}}$ mode while a $\mathrm{U}_{\mathrm{CW}} \mathrm{S}_{\mathrm{PS}}$ of different amplitude exists for the same value of $\varepsilon_{T}$. In the example illustrated in Fig. 7 the parameters are $\varepsilon_{\alpha}=1, \varepsilon_{X}=0.1, \mu=M=0.1$, $\alpha=A=0.1, \kappa_{\alpha}=0.1, \kappa_{X}=0.1, E=0.1$ and $N=39$. For $\varepsilon_{T}=15$ and $\varepsilon_{T}=5$ there exist $\mathrm{CW}$ nonlinear modes that fall respectively in these two cases as shown in the bifurcation diagram, Fig. 7(a). In Fig. 7(b) the asymptotic behavior of the chosen CW mode shows that there is a sharp transition to a stable $\mathrm{CW}$ mode on the $\mathrm{S}_{\mathrm{CW}}$ branch. Sharp transition towards the respective value on the $\mathrm{U}_{\mathrm{CW}} \mathrm{S}_{\mathrm{PS}}$ branch also occurs for the second choice of $\varepsilon_{T}$ as shown in Fig. 7(c). However, the asymptotic 


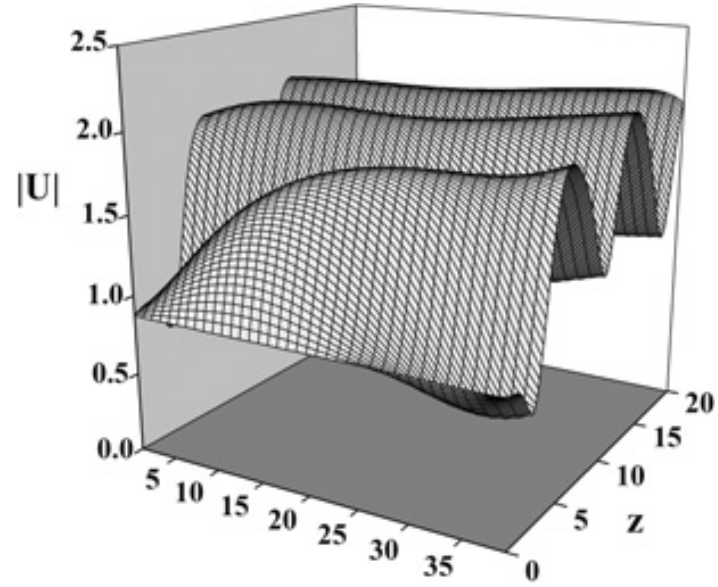

(a)

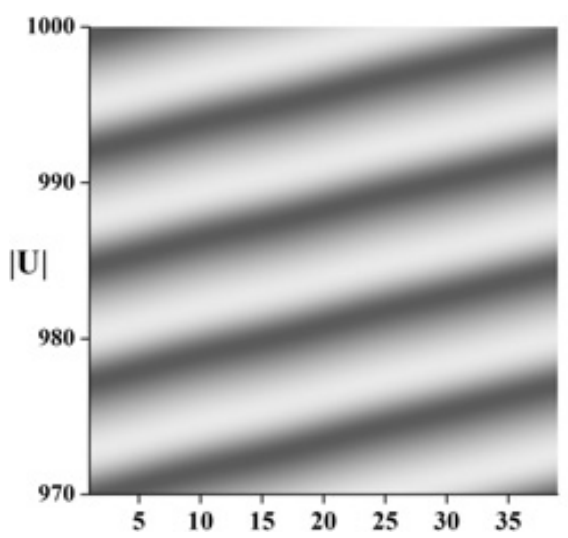

$\mathbf{U}_{\mathrm{cw}} \mathbf{S}_{\mathrm{ps}}$

(c)

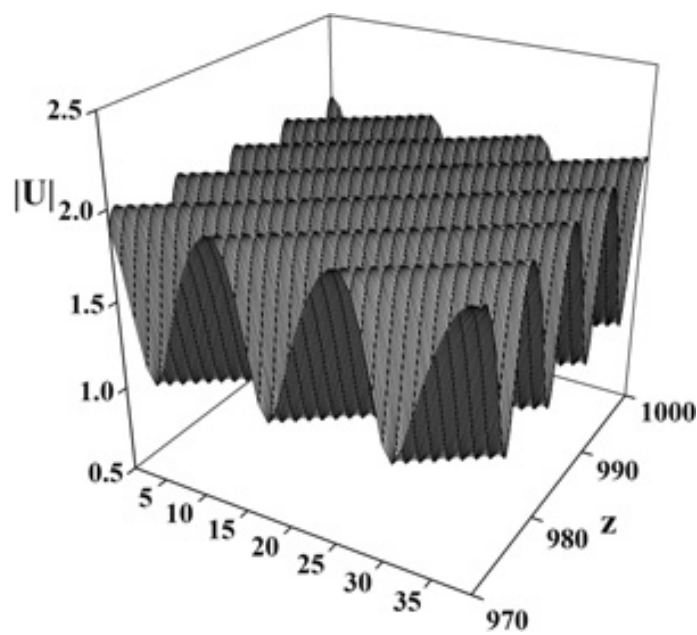

(e)

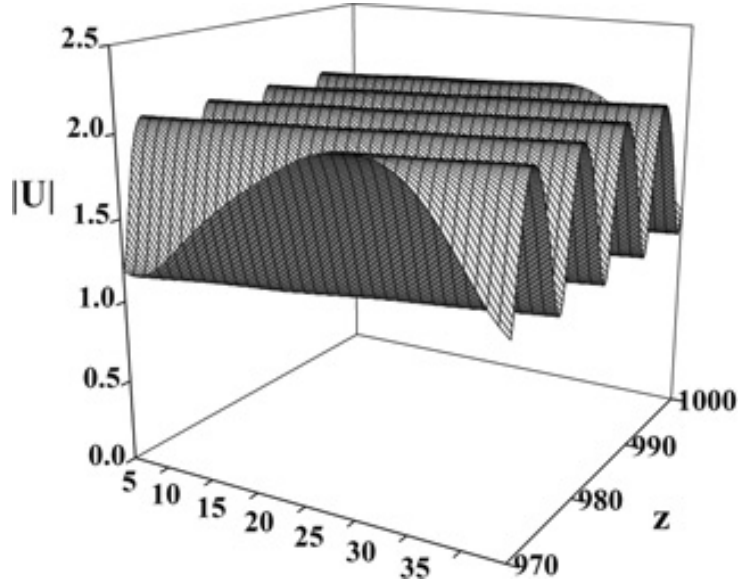

(b)

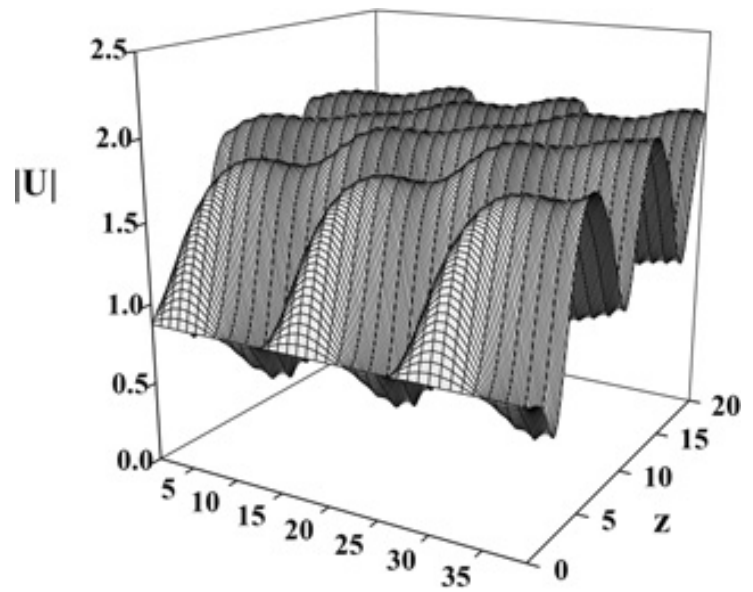

(d)

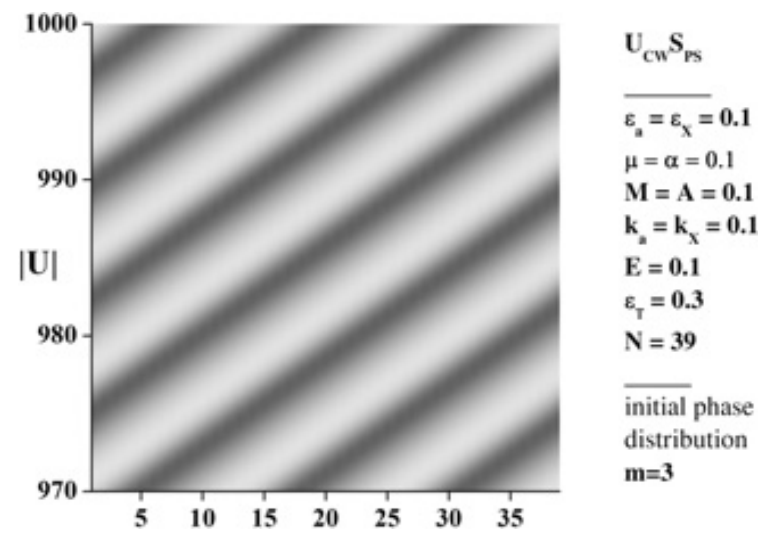

(f)

Fig. 6. $\mathrm{U}_{\mathrm{CW}} \mathrm{S}_{\mathrm{PS}}$ mode (all nodes are set at $z=0$ to their respective $\mathrm{CW}$ values) phase modulated through a phase factor $\exp (j 2 \pi n m / N)$ with $\varepsilon_{\alpha}=1, \varepsilon_{X}=0.1, \mu=M=0.1, \alpha=A=0.1, \kappa_{\alpha}=0.1, \kappa_{X}=0.1, E=0.1, \varepsilon_{T}=0.3$ and $N=39$. (a)-(c): short $z$-behavior, asymptotic behavior in three-dimensional and gray scale plot with $m=1$, (d)-(f): short $z$-behavior, asymptotic behavior in three-dimensional and gray scale plot with $m=3$. 


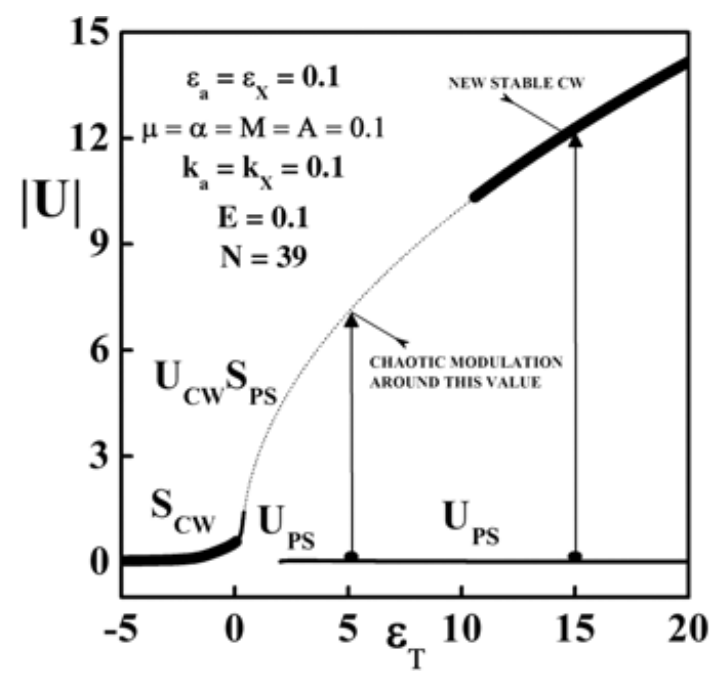

(a)

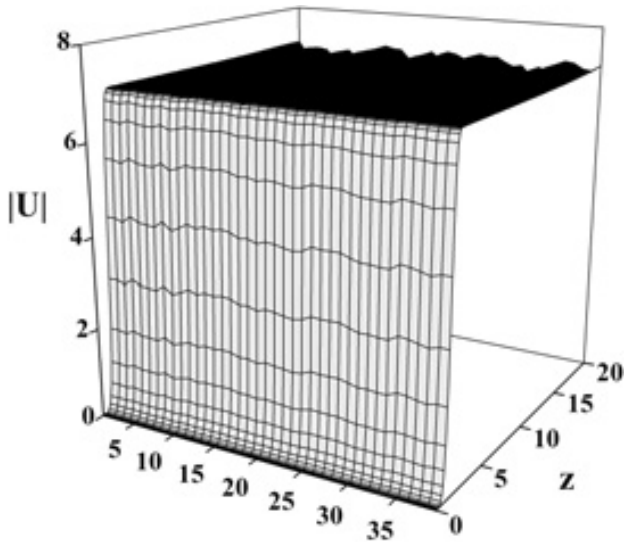

(c)

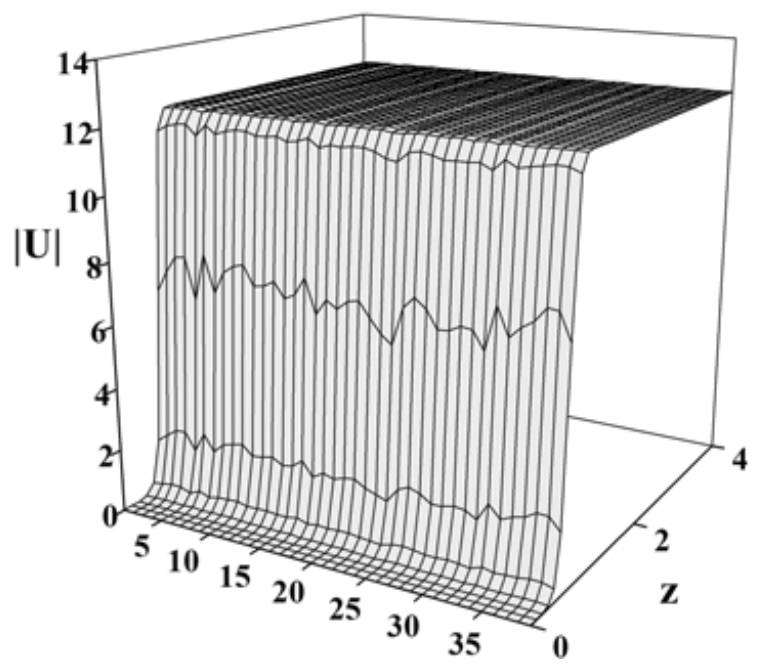

$\mathbf{U}_{\mathrm{PS}}$

$\varepsilon_{\mathrm{a}}=\varepsilon_{\mathrm{x}}=0.1$

$\mu=\alpha=0.1$

$\mathrm{M}=\mathrm{A}=\mathbf{0 . 1}$

$\mathrm{k}_{\mathrm{a}}=\mathrm{k}_{\mathrm{x}}=0.1$

$\mathrm{E}=0.1$

$\varepsilon_{\mathrm{T}}=15$

$\mathrm{N}=39$

random initial perturbation

(b)

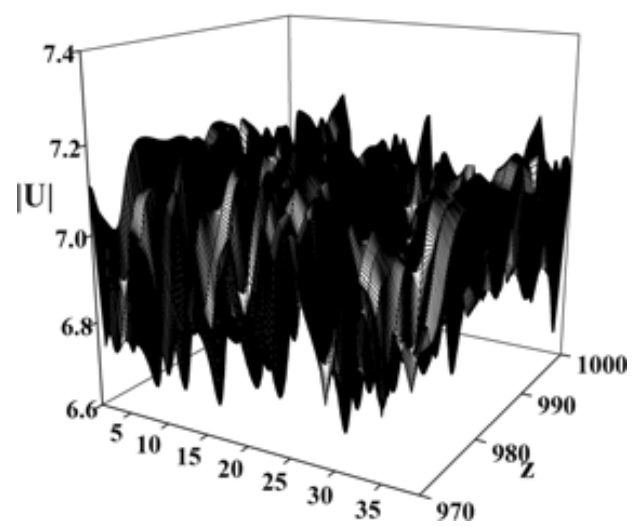

(d)

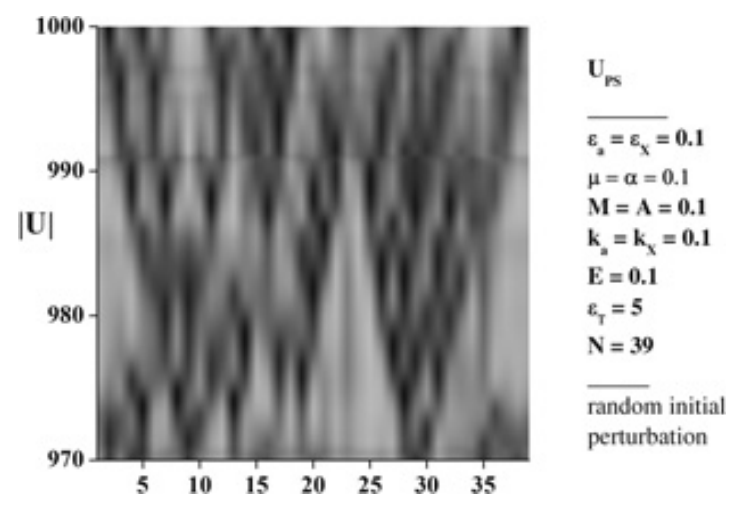

(e)

Fig. 7. (a) Bifurcation diagram for $\varepsilon_{\alpha}=1, \varepsilon_{X}=0.1, \mu=M=0.1, \alpha=A=0.1, \kappa_{\alpha}=0.1, \kappa_{X}=0.1, E=0.1$ and $N=39$; (b) $\mathrm{U}_{\mathrm{PS}}$ mode at $\varepsilon_{T}=15$ exhibiting fast transition to a $\mathrm{S}_{\mathrm{CW}}$ of different amplitude (all nodes are set at $z=0$ to their respective $\mathrm{CW}$ values); (c)-(e) short $z$-behavior, asymptotic behavior in three-dimensional and gray scale plot for $\mathrm{U}_{\mathrm{PS}}$ mode at $\varepsilon_{T}=5$ with a $\mathrm{U}_{\mathrm{CW}} \mathrm{S}_{\mathrm{PS}}$ of different amplitude existing for the same value of $\varepsilon_{T}$ (all nodes are set at $z=0$ to their respective $\mathrm{CW}$ values). 


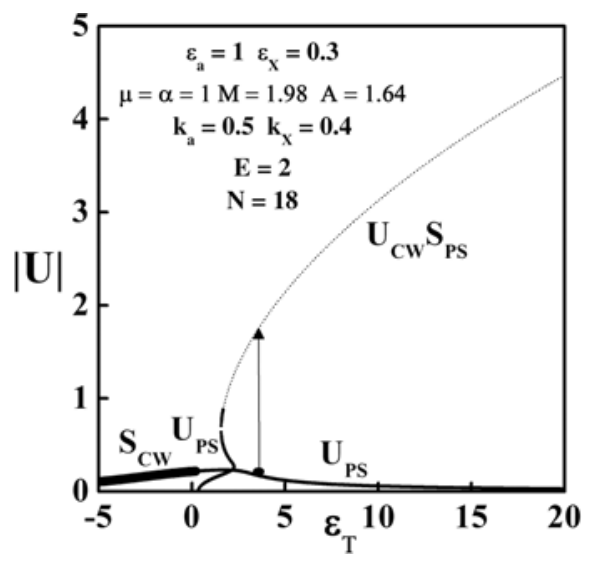

(a)

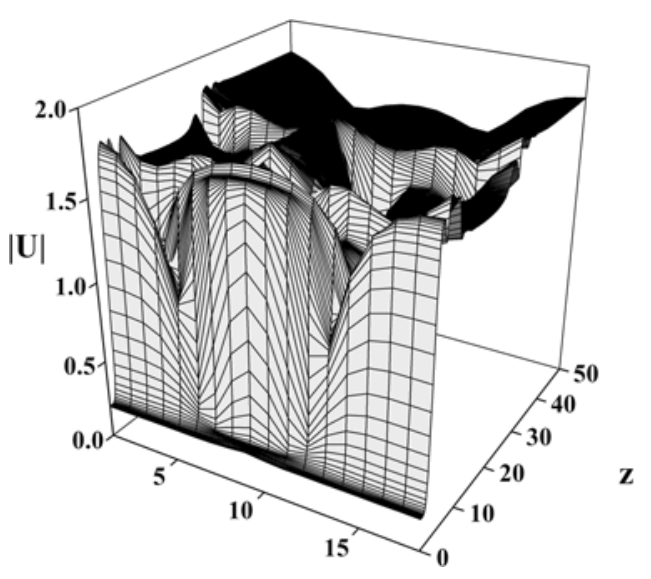

(b)

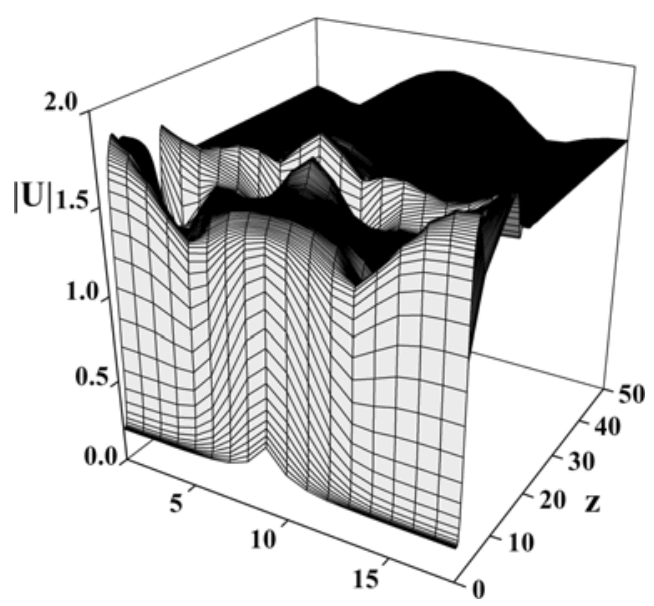

(d)

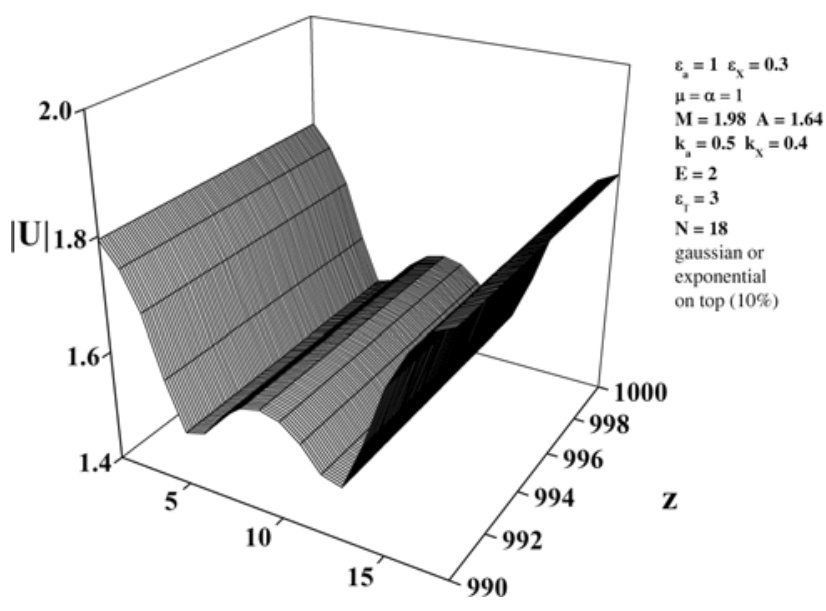

(c)

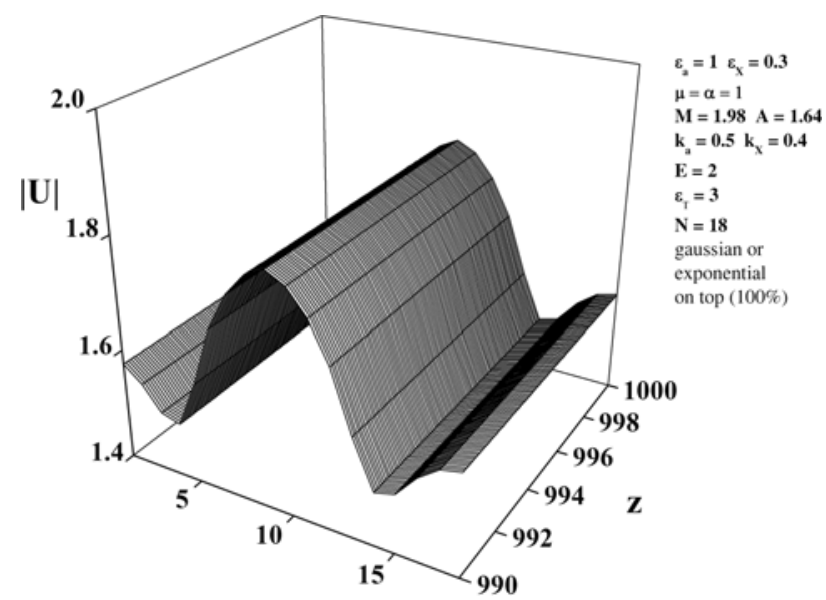

(e)

Fig. 8. (a) Bifurcation diagram for $\varepsilon_{\alpha}=1, \varepsilon_{X}=0.3, \mu=\alpha=1, M=1.98, A=1.64, \kappa_{\alpha}=0.5, \kappa_{X}=0.4, E=2, \varepsilon_{T}=3$ and $N=22$. A $U_{P S}$ mode with a $U_{C W} S_{P S}$ of different amplitude existing for the same value of $\varepsilon_{T}$ (all nodes are set at $z=0$ to their respective CW values) is chosen. (b)-(c) a localized (gaussian or exponential) amplitude perturbation of $10 \%$ is applied on top of the unstable CW mode; (d)-(e) a localized (gaussian or exponential) amplitude perturbation of $100 \%$ is applied on top of the unstable CW mode. (b) and (d) transitional behavior; (c) and (e) asymptotic behavior. 
behavior of this mode is more or less chaotic as one may easily see in both the three-dimensional and the gray scale plots of Figs. 7(d) and 7(e). The amplitude fluctuates erratically around the respective value on the $\mathrm{U}_{\mathrm{CW}} \mathrm{S}_{\mathrm{PS}}$ branch.

The aforementioned behavior of a $\mathrm{U}_{\mathrm{PS}} \mathrm{CW}$ nonlinear mode underneath a $\mathrm{U}_{\mathrm{CW}} \mathrm{S}_{\mathrm{PS}}$ of different amplitude at the same value of $\varepsilon_{T}$ can be drastically altered if one tailors in a controllable way the input: Setting all nodes of the circular array and the central one at $z=0$ to the respective unstable mode values and imposing on top a localized (irrespectively gaussian or exponential) amplitude perturbation the system locks at a higher localized (in $n$ ) and stationary (not varying with $z$ ) mode that is, a solitary type nonlinear mode. In Fig. 8 the parameters are $\varepsilon_{\alpha}=1, \varepsilon_{X}=0.3, \mu=\alpha=1$, $M=1.98, A=1.64, \kappa_{\alpha}=0.5, \kappa_{X}=0.4, E=2$, $\varepsilon_{T}=3$ and $N=22$. In Fig. 8(a) the respective bifurcation diagram and the transition is shown. In Figs. 8(b) and 8(c) the localized (gaussian or exponential) amplitude perturbation is $10 \%$ on top of the unstable CW mode, while in Figs. 8(d)-8(e) the perturbation is $100 \%$. Both the transitional [Figs. 8(b) and $8(\mathrm{~d})$ ] and the asymptotic [Figs. 8(c) and 8(e)] behavior are shown. It is, therefore, evident that perturbing controllably and locally an unstable to periodic perturbations $\mathrm{CW}$ nonlinear mode leads to the appearance of solitary type structures, although the localization is rather weak.

\section{Conclusions}

The investigation focused on the existence of stable localized solutions and pattern formation in centrally coupled circular arrays. In the model under investigation all the nonlinear coupling coefficients were complex, that is nonlinear losses were taken into account. In such a case, at least when the central coupling is absent, confined discrete solitarytype structures may exist. This expectation is further strengthened because there is evidence in the literature that breathing solutions for the discrete Ginzburg-Landau equation (which is the planar-periodic no-central element limit of the models considered in this work) do exist. Our investigation thus focused on the existence of breathing pattern solutions, that is, solutions whose intensity exhibit azimuthally periodic behavior as the light propagates along $z$. While the stability of the zero solution is of great importance for the investigation of the existence of stable localized modes in open systems (in the unstable situation the optical power may concentrate on a subset of nodes leading to solitary and/or breather type of structure), this is not the case in the circular system in hand. The reason is that localized structures residing on an open (linear) array have their intensity dropping to zero at their tails, while in a circular system the tails coincide.

The investigation concentrated on the existence and stability (to amplitude modulations and/or periodic perturbations) of nonlinear CW modes. The regions and character of stability of these nonlinear modes span a wide region in the parameter space which extends beyond the linear stability region. Respective bifurcation diagrams reveal a multitude of branches of various kinds of stability/instability situations. Exploiting these stability and bifurcation diagrams proves a quite effective tool in our investigation whose results may briefly be summarized as follows:

- Setting all nodes of the circular array and the central one at $z=0$ to the respective $\mathrm{U}_{\mathrm{CW}} \mathrm{S}_{\mathrm{PS}}$ mode values multiplied by an exponential or gaussian $\left[\exp \left(-|n-n 0|^{\nu}\right)(\nu=1,2)\right]$ and the central one set at its respective $\mathrm{U}_{\mathrm{CW}} \mathrm{S}_{\mathrm{PS}}$ value, a periodic mode is established after a transition period.

- Setting all nodes of the circular array and the central one at $z=0$ to the respective $\mathrm{U}_{\mathrm{CW}} \mathrm{S}_{\mathrm{PS}}$ mode values and perturbing them randomly (amplitude and phase) the same (irrespectively of the initial perturbation) periodic mode is established after a transition period.

- Setting all nodes of the circular array and the central one at $z=0$ to the respective $\mathrm{U}_{\mathrm{CW}} \mathrm{S}_{\mathrm{PS}}$ mode values and modulating each node through a phase factor a fully controllable periodic mode is established after a very short transition period.

- Setting all nodes of the circular array and the central one at $z=0$ to the respective values of a $\mathrm{U}_{\mathrm{PS}}$ mode lying under a $\mathrm{S}_{\mathrm{CW}}$ mode and perturbing them randomly (amplitude and phase) the system locks at the higher $\mathrm{S}_{\mathrm{CW}}$ mode.

- Setting all nodes of the circular array and the central one at $z=0$ to the respective values of a $U_{P S}$ mode located under a $U_{C W} S_{P S}$ mode and perturbing them randomly (amplitude and phase) the $U_{C W} S_{P S}$ mode is excited, though with a chaotic modulation that persists. If, instead of randomly perturbing it, one imposes on top of the chosen CW a localized (gaussian or exponential) amplitude perturbation the system locks at 
a higher adequately localized (in $n$ ) and stationary (in $z$ ) mode of solitary type.

These properties render the concept ideal for the design of high power stable amplifiers as well as of all-optical data processing devices in optical communications since it is functionally rich, far more controllable than a planar one and easier to stabilize.

\section{Acknowledgment}

This work was supported by the Greek Ministry of Education through the Heracletos and Pythagoras grants.

\section{References}

Akhemediev, N. N. \& Ankiewitz, A. [1977] Solitons, Nonlinear Pulses and Beams (Chapman and Hall, London).

Anderson, B. P. \& Kasevich, M. A. [1998] "Macroscopic quantum interference from atomic tunnel arrays," Science 282, 1686-1689.

Aranson, I. S. \& Kramer, L. [2002] "The world of the complex Ginzburg-Landau equation," Rev. Mod. Phys. 74, 99-143.

Arecchi, F. T., Bragard, J. \& Castelano, L. M. [2002] Bose-Einstein Condensates and Atom Lasers, eds. Martellucci, S., Chester, A. N., Aspect, A. \& Inguscio, M. (Kluwer, NY).

Christodoulides, D. N. \& Joseph, R. I. [1988] "Discrete self-focusing in nonlinear arrays of coupled waveguides," Opt. Lett. 13, 794-796.

Cross, M. C. \& Hohenberg, P. C. [1993] "Pattern formation outside of equilibrium," Rev. Mod. Phys. 65, 851-1112.

Desurvire, E. [1994] Erbium-Doped Fiber Amplifiers: Principles and Applications (John Wiley, NY).

Efremidis, N. \& Hizanidis, K. [2002] "Complexcubic Ginzburg-Landau equation-based model for erbium-doped fiber-amplifier-supported nonreturnto-zero communications," J. Opt. Soc. Am. B 19, $63-74$.

Efremidis, N. K., Hudock, J. \& Christodoulides, D. N. [2002] "Discrete solitons in nonlinear zigzag waveguide arrays with tailored diffraction properties," paper NLMD35, Nonlinear Guided Waves and their Applications, Stresa, Italy, 1-4 September 2002.

Efremidis, N. K. \& Christodoulides, D. N. [2003] "Discrete Ginzburg-Landau solitons," Phys. Rev. E 67, 026606 .

Eisenberg, H. S. et al. [1998] "Discrete spatial optical solitons in waveguide arrays," Phys. Rev. Lett. 81, 3383-3386.

Hocking, L. M. \& Stewartson, K. [1992] "On the nonlinear response of a marginally unstable plain parallel flow to a two-dimensional disturbance," Proc. R. Soc. London, Ser. A 326, 289-313.

Kittel, C. [1986] Introduction to Solid State Physics (Wiley, NY).

Kneer, B., Wong, T., Vogel, K., Schleich, W. P. \& Walls, D. F. [1998] "Generic model of an atom laser," Phys. Rev. A 58, 4841-4853.

Kuramoto, Y. [1984] Chemical Oscillations, Waves and Turbulence (Springer, Berlin).

Malomed, B. A. \& Winful, H. G. [1984] Phys. Rev. E 53, 1581.

Manneville, P. [1990] Dissipative Structures and Weak Turbulence (Academic, San Diego).

Maruno, K., Ankiewicz, A. \& Akhmediev, N. [2003] "Exact localized and periodic solutions of the discrete complex Ginzburg-Landau equation," Opt. Commun. 221, 199-209.

Nozaki, K. \& Bekki, N. [1984] "Exact solution of the generalized Ginzburg-Landau equation," J. Phys. Soc. Jpn. 53, 1581-1582.

Otsuka, K. [1999] Nonlinear Dynamics in Optical Complex Systems (KTK Scientific Publishers, Tokyo).

Pereira, N. R. \& Stenflo, L. [1977] "Nonlinear Schrödinger equation including growth and damping," Phys. Fluids 20, 1733-1734.

Schmidt-Hattenberger, C., Trutschel, U., Muschall, R. \& Lederer, F. [1991] "Envelope description of an optical fibre array with circularly distributed multiple cores," Opt. Commun. 82, 461-465.

Wang, S. S. \& Winful, H. G. [1988] "Dynamics of phaselocked semiconductor laser arrays," Appl. Phys. Lett. 52, 1774-1776.

Willaime, H., Cardoso, O. \& Tabeling, P. [1991] "Frustration in a linear array of vortices," Phys. Rev. Lett. 67, 3247-3250. 